CORRIGENDUM

Corrigendum: Scalar radiation emitted from a source rotating around a black hole (2000 Class. Quantum Grav. 17 19)

To cite this article: L C B Crispino et al 2016 Class. Quantum Grav. 33209502

View the article online for updates and enhancements.
Related content

Corrigenda P.J. McCarthy, K.S. Riedel, O.J.W.F. Kardaun et al.

- Corrigenda

B.B. Kadomtsev

- Corrigendum

L.M. Kovrizhnykh, S.G. Shasharina and Yu.A. Volkov

\section{Recent citations}

$$
\begin{aligned}
& \text { - Scalar radiation from a radially infalling } \\
& \text { source into a Schwarzschild black hole in } \\
& \text { the framework of quantum field theory } \\
& \text { Leandro A. Oliveira et al }
\end{aligned}
$$

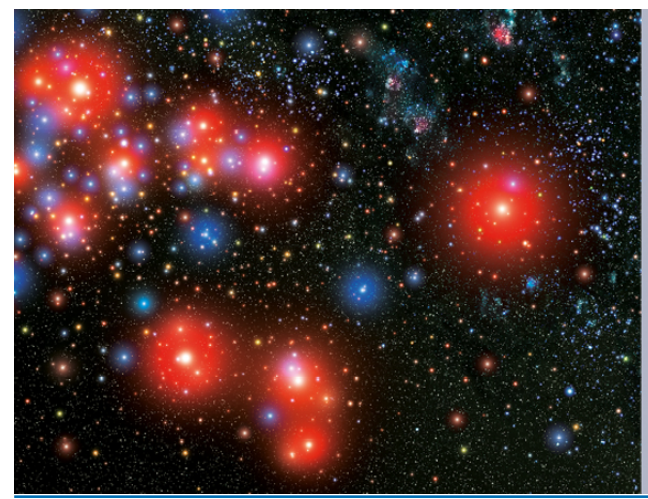

A.A

Part of your publishing universe and your first choice for astronomy, astrophysics, solar physics and planetary science ebooks. iopscience.org/books/aas 


\title{
Corrigendum: Scalar radiation emitted from a source rotating around a black hole (2000 Class. Quantum Grav. 17 19)
}

\author{
L C B Crispino ${ }^{1}$, A Higuchi ${ }^{2}$ and G E A Matsas ${ }^{3}$ \\ ${ }^{1}$ Faculdade de Física, Universiade Federal do Pará, 66075-110, Belém, Pará, Brazil \\ ${ }^{2}$ Department of Mathematics, University of York, Heslington, York YO10 5DD, UK \\ ${ }^{3}$ Instituto de Física Teórica, Universidade Estadual Paulista, Rua Dr. Bento Teobaldo \\ Frerraz 271, 01140-070, São Paulo, Brazil
}

E-mail: crispino@ufpa.br, atsushi.higuchi@york.ac.uk and matsas@ift.unesp.br

Received 22 June 2016

Accepted for publication 15 September 2016

Published 29 September 2016

Incorrect formulae were used in (35) and (36) for estimating the portion of the radiation emitted to infinity. The correction to these equations has very little effect on figure 5 , and does not change the conclusion that almost all radiation is emitted to infinity rather than into the black hole.

The power radiated to infinity should have been computed by coupling the source to the modes radiated to future infinity with no flux going into future horizon. These modes are related to those coming from past infinity with radial functions $\overleftarrow{\psi}{ }_{\omega l}^{S}(r)$ by time reversal. Thus, the power emitted to infinity is obtained by replacing these radial functions by their complex conjugates in the formula for finding the power $\overleftarrow{W}_{l m}^{S \text {, em }}$ (see (17)). Since complex conjugation does not affect the result - this invariance is due to the stationary nature of the orbit of the scalar source-the power emitted to infinity with fixed angular-momentum quantum numbers is simply $\overleftarrow{W}_{l m}^{S, \text { em }}$. Therefore, equations (35) and (36) should be replaced by

$$
W^{S, \mathrm{obs}}=\sum_{l=1 m=1}^{\infty} \sum_{W_{l m}}^{S, \mathrm{em}}
$$

The subsequent low-energy approximation for $W^{S \text {,obs }}$ needs to be corrected accordingly. We also note that similar corrections need to be made in [1-6], which used equations similar to (35) and (36) for estimating the portion of the radiation emitted to infinity.

It is interesting to note that $\psi_{\omega l}^{S *}(r)=\overleftarrow{\mathcal{R}}_{\omega l}^{*} \overleftarrow{\psi}_{\omega l}^{S}(r)+\overleftarrow{\mathcal{T}}_{\omega l}^{*} \vec{\psi}_{\omega l}^{S}(r)$ and hence

$\left|\overleftarrow{\psi_{\omega l}^{S}}(r)\right|^{2}=\left|\overleftarrow{\mathcal{R}}_{\omega l}\right|^{2}\left|\overleftarrow{\psi}_{\omega l}^{S}(r)\right|^{2}+\left|\overleftarrow{\mathcal{T}}_{\omega l}\right|^{2}\left|\vec{\psi}_{\omega l}^{S}(r)\right|^{2}+2 \operatorname{Re}\left[\overleftarrow{\mathcal{R}}_{\omega l}^{*} \overleftarrow{\mathcal{T}}_{\omega l} \overleftarrow{\psi}_{\omega l}^{S}(r) \vec{\psi}_{\omega l}^{S *}(r)\right]$

Since $\overleftarrow{\mathcal{T}}_{\omega l}=\overrightarrow{\mathcal{T}}_{\omega l}$, the error corresponded to missing the interference term in this equation. 
The authors thank Vitor Cardoso and Leandro Oliveira for raising questions that led to this correction.

\section{References}

[1] Castiñeiras J, Crispino L C B, Murta R and Matsas G E A 2005 Braz. J. Phys. 351080

[2] Castiñeiras J, Crispino L C B, Murta R and Matsas G E A 2005 Phys. Rev. D 71104013

[3] Castiñeiras J, Crispino L C B and Meira Filho D P 2007 Phys. Rev. D 75024012

[4] Crispino L C B 2008 Phys. Rev. D 77047503

[5] Crispino L C B, da Silva A R R and Matsas G E A 2009 Phys. Rev. D 79024004

[6] Macedo C F B, Crispino L C B and Cardoso V 2012 Phys. Rev. D 86204002 